\title{
Comparison of the Economics and Performance of Horizontal and Vertical Wells
}

\author{
O. K. Dankwa ${ }^{1 *}$, S. N. Gomah Ahmed ${ }^{2}$ and P. Opoku Appau ${ }^{3}$ \\ ${ }^{I}$ Department of Petroleum Engineering, University of Mines and Technology, P. O. Box, 237, Tarkwa \\ ${ }^{2}$ Department of Petroleum Product Marking Scheme, National Petroleum Authority, Accra, Ghana \\ ${ }^{3}$ Research Institute of Enhanced Oil Recovery, China University of Petroleum (Beijing), P. R. China
}

*Corresponding Author: O. K. Dankwa, Department of Petroleum Engineering, University of Mines and Technology, Tarkwa

\begin{abstract}
Horizontal wells generally have a better performance than vertical wells in terms of productivity, but are however more expensive to drill, complete and produce from. This work compares the performance of the two types of wells by estimating Productivity Index (PI) for horizontal and vertical wells under same reservoir parameters and determines which of the two types of wells is more economical. Joshi and Borisov's models for predicting horizontal Productivity Index are adopted in this research, while vertical Productivity Index is predicted using Joshi's model only. Economic evaluations of the two wells are done using investment decision criteria known as the Net Present Value (NPV). Hypothetical data used in all calculations generated results that indicated that horizontal wells have higher productivity than the vertical wells, and that the horizontal wells in this project are more economically acceptable than the vertical wells. In conclusion, Horizontal well Productivity Index increases with increasing horizontal well length while vertical productivity index is not affected by well length. Both horizontal and vertical productivity indices increase with increasing reservoir thickness. It is recommended that subsequent work in this field of study should consider productivity models that account for pseudo-steady state flow and reservoir anisotropy.
\end{abstract}

Keywords: Economics, Horizontal and Vertical wells, Net Present Value (NPV), Productivity Index, Well Performance

\section{INTRODUCTION}

During the initial stages of well planning, engineers will have to make the choice between drilling a vertical well or a horizontal well for a specific type of reservoir with unique properties that either types of drilling methods could favor. A reservoir with good height, thickness and a very large surface area will require a specific well type suitable to effectively drain it. Faced with this challenge, well planners, drilling engineers, production engineers and reservoir engineers have to make a choice on whether to drill several vertical wells on the location or to drill just one horizontal well to satisfactorily cover the reservoir area for effective drainage.

Several factors will affect the choice of the options available; some of which are; economics, information of reservoir characteristics from seismic surveys such as reservoir shape, height, length and dip angle of the reservoir, expected monetary returns on investment based on the productivity of the wells drilled, etc. Well performance is often measured in terms of the well's productivity which is dependent on a number factors such as the reservoir's configuration, the type of completion, petrophysical and fluid properties, formation damage, etc [1]. The productivity index of a well is a function of the pressure losses between the reservoir boundary and the well bore [2-5]. The factors that affect productivity index are reservoir drainage area, pay zone thickness, anisotropy, well length, fluid velocity, and well completion methods [6-8].

A vertical well is a well that is characterized by a generally vertical wellbore track. Because the risk of vertical well construction is relatively low, the techniques for drilling such a well are relatively simple and the maintenance of the subsequent oil extraction operation is relatively easy. Vertical well is the most widely used well type worldwide $[9,10]$. In horizontal wells, the wellbore remains in high angle trajectory roughly parallel to the formation, thereby exposing significantly more attention zone 
to production than would be exposed by a vertical well [11]. Productivity index for horizontal wells increases with well length, and anisotropy value; also horizontal wells are better united for thin beds $[12,13]$. This study seeks to determine the productivity and economic viability of a vertical and horizontal well for specific reservoir conditions in order to select the more suitable well type for the prevailing reservoir conditions.

\section{MATERIALS AND MeTHOdS}

The model employed in this research to predict the productivity of the vertical wells was Joshi's method. Horizontal well productivity predictions in this project have been done by adopting two models which include Joshi's model and the Borisov's model. Reasonable reservoir, fluid, and well data were assumed for the prediction of both vertical and horizontal well productivity indices.

Net Present Value (NPV) is the commonest investment decision criteria used to access the economic viability of an Exploration and Production (E\&P) venture in today's market. It is one of the many criteria that takes into account the time value of money and is relatively simpler to use than other decision criteria.

\subsection{Horizontal Productivity Models}

\section{Borisov's Model}

Borisov in 1984 proposed the following expression for predicting the productivity index of a horizontal well in an isotropic reservoir [14], i.e., $\mathrm{k}_{\mathrm{v}}=\mathrm{k}_{\mathrm{h}}$

$$
J_{h}=\frac{0.00708 h k_{h}}{\mu_{o} \mathrm{~B}_{o}\left[\ln \left(\frac{4 r_{e h}}{L}\right)+\left(\frac{h}{L}\right) \ln \left(\frac{h}{2 \pi r_{w}}\right)\right]}
$$

Where, $\mathrm{J}_{\mathrm{h}}=$ horizontal productivity index, $\mathrm{STB} /$ day/psi

$\mathrm{kh}=$ horizontal permeability, $\mathrm{md}$

$\mathrm{h}=$ thickness, $\mathrm{ft}$.

$\mathrm{L}=$ well length

Bo $=$ oil formation volume factor, bbl/STB

$\mu_{\mathrm{o}}=$ oil viscosity, $\mathrm{cp}$.

$\mathrm{r}_{\mathrm{eh}}=$ drainage radius, $\mathrm{ft}$.

$\mathrm{r}_{\mathrm{w}}=$ wellbore radius, $\mathrm{ft}$

\section{Joshi's Model}

Joshi in 1991 presented the following expression for estimating the productivity index of a horizontal well in isotropic reservoirs [15]. Joshi accounted for the influence of the reservoir anisotropy by introducing the vertical permeability kv into his equation.

$$
J_{h}=\frac{0.00708 h k_{h}}{\mu_{o} \mathrm{~B}_{o}\left[\ln (R)+\left(\frac{B^{2} h}{L}\right) \ln \left(\frac{h}{2 r_{w}}\right)\right]}
$$

Where, $\mathrm{J}_{\mathrm{h}}=$ horizontal productivity index, $\mathrm{STB} /$ day/psi

$\mathrm{k}_{\mathrm{h}}=$ horizontal permeability, md

$\mathrm{h}=$ thickness, $\mathrm{ft}$.

$\mathrm{L}=$ well length

$\mathrm{Bo}=$ oil formation volume factor, $\mathrm{bbl} / \mathrm{STB}$

$\mu_{\mathrm{o}}=$ oil viscosity, $\mathrm{cp}$.

$\mathrm{r}_{\mathrm{w}}=$ wellbore radius, $\mathrm{ft}$

The parameters R and B are represented by the equations below, 


$$
R=\frac{a+\sqrt{a^{2}-(L / 2)^{2}}}{(L / 2)}
$$

Where, $\mathrm{L}=$ well length

$\mathrm{a}=$ half the major axis of drainage of the ellipse and expressed by the equation below.

$a=\left(\frac{L}{2}\right)\left[0.5+\sqrt{0.25+\left(2 r_{e h} / L\right)^{4}}\right]^{0.5}$

$B=\sqrt{\frac{k_{h}}{k_{v}}}$

Where, $\mathrm{k}_{\mathrm{h}}=$ horizontal permeability, md

$\mathrm{k}_{\mathrm{v}}=$ vertical permeability, md

$\mathrm{r}_{\text {eh }}=$ drainage radius, $\mathrm{ft}$.

\section{Joshi's Method for Drainage Area}

Figure 1 shows the drainage areas for a vertical well (Fig 1a) and a horizontal well (Fig. 1b). A vertical well drains a cylindrical volume as illustrated while a horizontal well drains an ellipsoid. It is generally expected that a horizontal well drains a larger volume than a vertical well [15].
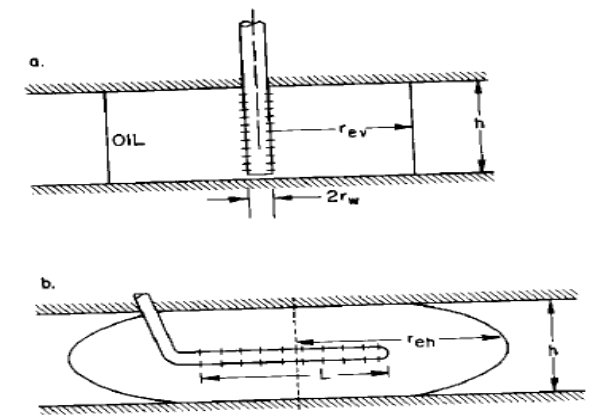

Figure1. A Schematic of a Vertical and Horizontal Well Drainage Area (Joshi, 1991)

A horizontal well can be looked upon as a number of vertical wells drilling next to each other and completed in a limited pay zone thickness. Figure 2 below shows the drainage area of a horizontal well of length (L) in a reservoir with a pay zone thickness (h). Each end of the horizontal well would drain a half-circular area of radius $b$, with a rectangular drainage shape of the horizontal well [16].

Joshi (1991) assumed that each end of the horizontal well is represented by a vertical well that drains an area of a half circle with a radius of $b$. He therefore proposed the following two methods for calculating the drainage area of a horizontal well.

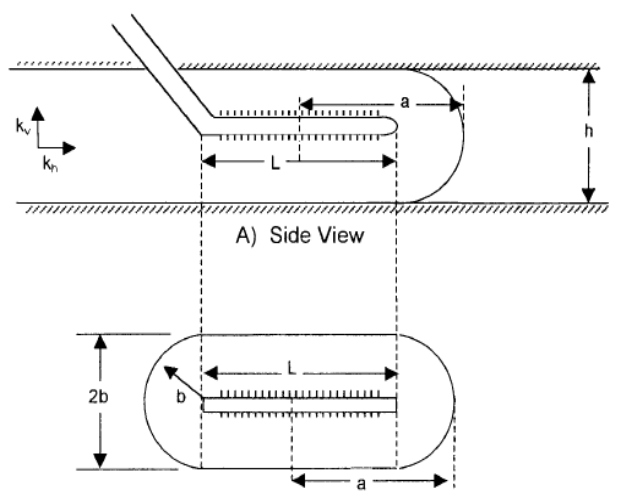

B) Top View

Figure2. Schematic of a Horizontal Well Drainage Area (Tarek, 2006) 
Method 1: Under this method, Joshi proposed that the drainage area is represented by two half circles of radius $b$ (equivalent to a radius of a vertical well rev) at each end and a rectangle, of dimensions $\mathrm{L}$ $(2 \mathrm{~b})$, in the center. The drainage area is therefore represented by the equation below.

$$
A=\frac{L(2 b)+\pi b^{2}}{43560}
$$

Where, $\mathrm{A}=$ drainage area, acres

$\mathrm{L}=$ length of horizontal well, $\mathrm{ft}$.

$\mathrm{b}=$ half minor axis of an ellipse, $\mathrm{ft}$.

Method 2: In method 2, Joshi assumed that total horizontal drainage area is an ellipse and is represented by the equation below.

$$
A=\frac{\pi a b}{435600}
$$

Where, $\mathrm{A}=$ drainage area

$\mathrm{a}=$ major axis of an ellipse also represented mathematically as shown below

$a=\frac{L}{2}+b$

Where, $\mathrm{L}=$ well length, ft.

$\mathrm{b}=$ half minor axis of an ellipse, $\mathrm{ft}$.

Joshi noted that the two methods give different values for the drainage area A and suggested assigning the average value for the drainage of the horizontal well.

Most methods used to predict productivity index of horizontal wells usually require a parameter known as drainage radius. The drainage radius of a well is represented mathematically as shown below

$$
r_{e h}=\sqrt{\frac{43560 A}{\pi}}
$$

Where, reh $=$ drainage radius, $\mathrm{ft}$.

$\mathrm{A}=$ reservoir area, acres

\subsection{Vertical Well Productivity}

Ideally bottom hole flowing pressure (Pwf) for at a certain flow rate (q) is measured using a bottomhole pressure gauge. A build up or drawdown test is used to estimate the average reservoir pressure (Pr) along with other parameters such as skin factor (s). Flow rate of a well producing under steady-state radial flow is given by:

$$
Q_{o}=\frac{0.00708 K_{o} h\left(P_{r}-P_{w f}\right)}{\mu_{o} B_{o}\left[\ln \left(\frac{r_{e}}{r_{w}}\right)+S\right]}
$$

Where, $\mathrm{Q}_{0}=$ oil flow rate, $\mathrm{STB} /$ day

$\mathrm{k}=$ permeability, md

$\mathrm{h}=$ thickness, $\mathrm{ft}$.

$\mathrm{s}=$ skin factor

$\mathrm{B}_{\mathrm{o}}=$ oil formation volume factor, bbl/STB

$\mu_{\mathrm{o}}=$ oil viscosity, $\mathrm{cp}$.

$\mathrm{Pi}=$ initial reservoir pressure, $\mathrm{psi}$

Pwf $=$ bottom hole flowing pressure, $\mathrm{psi}$ 
$\mathrm{r}_{\mathrm{e}}=$ reservoir radius, $\mathrm{ft}$.

$\mathrm{r}_{\mathrm{w}}=$ wellbore radius, $\mathrm{ft}$.

The productivity index of well can therefore be expressed as below:

$$
J=\frac{0.00708 k_{h}}{\mu_{o} B_{o}\left[\ln \left(\frac{r_{e}}{r_{w}}\right)+S\right]}
$$

Where, $\mathrm{J}=$ productivity index $(\mathrm{STB} / \mathrm{day} / \mathrm{psi})$

$\mathrm{k}_{\mathrm{h}}=$ horizontal permeability $(\mathrm{md})$

$\mu_{\mathrm{o}}=$ oil viscosity (cp)

$\mathrm{B}_{\mathrm{o}}=$ oil formation volume factor, bbl/STB

$\mathrm{r}_{\mathrm{e}}=$ reservoir radius, $\mathrm{ft}$.

$\mathrm{r}_{\mathrm{w}}=$ wellbore radius, $\mathrm{ft}$

$\mathrm{S}=$ skin factor

The skin factor is denoted by ' $\mathrm{S}$ ', and is expressed as;

$$
S=\left[\frac{k}{k_{\text {skin }}}-1\right] \ln \left(\frac{r_{\text {skin }}}{r_{w}}\right)
$$

Where, $\mathrm{S}=$ skin factor

$\mathrm{k}=$ reservoir permeability, md

$\mathrm{k}_{\mathrm{skin}}=$ permeability of damaged zone, $\mathrm{md}$

$\mathrm{r}_{\text {skin }}=$ radius of damaged zone, $\mathrm{ft}$

$\mathrm{r}_{\mathrm{w}}=$ wellbore radius, $\mathrm{ft}$

\subsection{NPV Calculation for the Horizontal and Vertical Well}

Using the productivity index value from Borisov's model with the well length of $1000 \mathrm{ft}$ and reservoir thickness of $160 \mathrm{ft}$, the net present value was calculated. Formulae used;

$d=1-\exp (-a)$

Where, $\mathrm{d}=$ rate of decline

$\mathrm{a}=$ effective annual decline

$a=\left[\ln \left(\frac{q_{i}}{q_{o}}\right)\right] / t$

Where, $\mathrm{a}=$ rate of buildup

$\mathrm{q}_{\mathrm{i}}=$ initial production, $\mathrm{bbl} /$ day.

$\mathrm{q}_{\mathrm{o}}=$ production at time $\mathrm{t}, \mathrm{bbl} / \mathrm{day}$.

$\mathrm{t}=$ time, years

$q_{t}=q_{i}\left(e^{-a t}\right)$

Where, $\mathrm{qt}=$ flow rate a certain time, $\mathrm{bbl} / \mathrm{day}$

$\mathrm{qi}=$ initial flow rate, bbl/day

$\mathrm{a}=$ buildup rate 
$\mathrm{t}=$ time, years.

$N_{p}=\left(\frac{1}{a}\right)\left(q_{i}-q_{o}\right)(1 / t)$

Where, $\mathrm{Np}=$ production for a period

$\mathrm{a}=$ buildup

$\mathrm{qi}=$ initial flow rate, bbl/day

$\mathrm{qo}=$ production at time $\mathrm{t}, \mathrm{bbl} / \mathrm{day}$.

$\mathrm{t}=$ time, years.

Equations 2.8 to equation 2.11 above are the equations used for production forecasting to aid in the calculation of the net present value. The equation below is the summary of the definition of Net Present Value and served as a guiding principle for all the NPV calculations in this project.

$$
N P V=\sum P V \text { of cash inflow @i* } \sum \sum V_{\text {of cash outflow @i* }}
$$

Where, NPV $=$ Net Present Value

$\mathrm{PV}=$ Present Value

$i^{*}=$ minimum rate of return

\section{RESUltS AND DisCUSSION}

\subsection{Results}

All hypothetical parameters that were used for productivity index calculations and all related calculations are displayed in Table 1. The productivity of the wells was calculated using Microsoft Excel software. The results of productivity index using Borisov and Joshi's models are summarized in Table 2 and Table 3 respectively. Figure 3 is a graph representing such a plot thus productivity ratio against well length.

The Tables 4 and 5 are a summary of computations of productivity index using Borisov and Joshi's models respectively. The resulting productivity indices were then plotted against the corresponding reservoir thickness as shown in Figure 4 below. Assumed data for all calculations of the Net present value done are displayed in table 6 below. The table 7, table 8, and table 9 shows summary of the calculations of net present value.

Table1. Parameters for Calculation of Productivity Index

\begin{tabular}{|l|l|l|l|l|l|}
\hline \multicolumn{2}{|c|}{ Reservoir dtata } & \multicolumn{2}{c|}{ Fluid data } & \multicolumn{2}{c|}{ Well data } \\
\hline Vertical Permeability, $\mathrm{K}_{\mathrm{v}}$ & $75 \mathrm{md}$ & Oil viscosity & $0.62 \mathrm{cp}$ & Well length, $\mathrm{L}$ & $1000 \mathrm{ft}$ \\
\hline Horizontal Permeability, $\mathrm{K}_{\mathrm{h}}$ & $75 \mathrm{md}$ & $\begin{array}{l}\text { Oil formation } \\
\text { volume factor, } \mathrm{B}_{\mathrm{o}}\end{array}$ & $\begin{array}{l}1.35 \\
\text { bbl/STB }\end{array}$ & Wellbore radius, $\mathrm{r}_{\mathrm{w}}$ & $0.365 \mathrm{ft}$ \\
\hline Reservoir thickness, $\mathrm{h}$ & $160 \mathrm{ft}$ & & & Reservoir radius, $\mathrm{r}_{\mathrm{e}}$ & $1053 \mathrm{ft}$ \\
\hline Porosity, $\phi$ & $3.8 \%$ & & & Area, A & $80 \mathrm{acres}$ \\
\hline & & & & Skin zone radius, $\mathrm{r}_{\mathrm{s}}$ & $1.312 \mathrm{ft}$ \\
\hline & & & $\begin{array}{l}\text { Permeability of skin } \\
\text { zone, } \mathrm{k}_{\text {skin }}\end{array}$ & $5.894 \mathrm{md}$ \\
\hline
\end{tabular}

Table2. Summary of Computations Using Borisov's Model

\begin{tabular}{|l|l|l|l|l|l|l|l|l|}
\hline Well length of horizontal section, ft. & $\mathbf{2 5 0}$ & $\mathbf{5 0 0}$ & $\mathbf{7 5 0}$ & $\mathbf{1 0 0 0}$ & $\mathbf{1 2 5 0}$ & $\mathbf{1 5 0 0}$ & $\mathbf{1 7 5 0}$ & $\mathbf{2 0 0 0}$ \\
\hline $\begin{array}{l}\text { Horizontal well productivity index, } \\
\text { STB/day/psi (Borisov's Model) }\end{array}$ & 18.32 & 29.09 & 38.57 & 47.94 & 57.73 & 68.33 & 80.14 & $\begin{array}{l}93.5 \\
9\end{array}$ \\
\hline $\begin{array}{l}\text { Vertical Productivity index, } \\
\text { STB/day/psi }\end{array}$ & 11.56 & 11.56 & 11.56 & 11.56 & 11.56 & 11.56 & 11.56 & $\begin{array}{l}11.5 \\
6\end{array}$ \\
\hline Productivity ratio & 1.58 & 2.52 & 3.34 & 4.15 & 4.99 & 5.91 & 6.93 & 8.09 \\
\hline
\end{tabular}

Table3. Summary of Computations Using Joshi's Model

\begin{tabular}{|l|c|c|c|c|c|c|c|c|}
\hline Well length of horizontal section, ft. & $\mathbf{2 5 0}$ & $\mathbf{5 0 0}$ & $\mathbf{7 5 0}$ & $\mathbf{1 0 0 0}$ & $\mathbf{1 2 5 0}$ & $\mathbf{1 5 0 0}$ & $\mathbf{1 7 5 0}$ & $\mathbf{2 0 0 0}$ \\
\hline $\begin{array}{l}\text { Horizontal well productivity index, } \\
\text { STB/day/psi (Joshi's Model) }\end{array}$ & 16.18 & 26.32 & 35.29 & 44.09 & 53.18 & 62.83 & 73.26 & 84.6 \\
\hline $\begin{array}{l}\text { Vertical Productivity index, } \\
\text { STB/day/psi }\end{array}$ & 11.56 & 11.56 & 11.56 & 11.56 & 11.56 & 11.56 & 11.56 & 11.5 \\
\hline \multicolumn{1}{|c|}{ Productivity ratio } & 1.40 & 2.28 & 3.05 & 3.81 & 4.60 & 5.43 & 6.34 & 7.32 \\
\hline
\end{tabular}




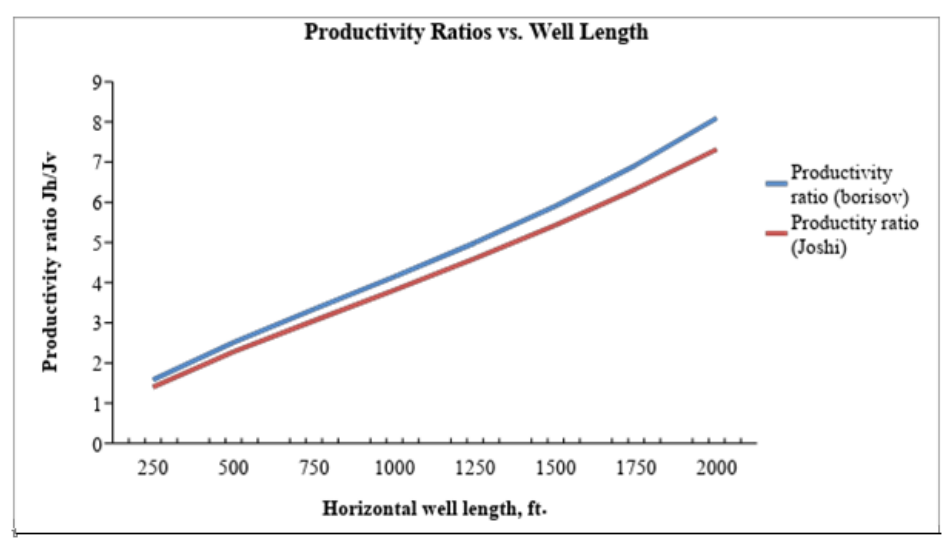

Figure3. Productivity Ratios Vs. Well Length

Table4. Productivity Index Values for Varying Reservoir Thickness

\begin{tabular}{|c|c|c|}
\hline Reservoir Thickness & Productivity Index (Borisov's Model) & Vertical Productivity Index \\
\hline 40 & 16.35 & 2.89 \\
\hline 50 & 19.92 & 3.61 \\
\hline 60 & 23.30 & 4.34 \\
\hline 70 & 26.47 & 5.06 \\
\hline 80 & 29.47 & 5.78 \\
\hline 90 & 32.29 & 6.50 \\
\hline 100 & 34.94 & 7.23 \\
\hline 110 & 37.44 & 7.95 \\
\hline 120 & 39.79 & 8.67 \\
\hline 130 & 42.01 & 9.39 \\
\hline 140 & 44.11 & 10.12 \\
\hline 150 & 46.08 & 10.84 \\
\hline 160 & 47.94 & 11.56 \\
\hline
\end{tabular}

Table5. Productivity Index Values for Varying Reservoir Thickness

\begin{tabular}{|c|c|c|}
\hline Reservoir Thickness & Productivity Index (Joshi's Model) & Vertical Productivity Index \\
\hline 40 & 15.86 & 2.89 \\
\hline 50 & 19.21 & 3.61 \\
\hline 60 & 22.33 & 4.34 \\
\hline 70 & 25.24 & 5.06 \\
\hline 80 & 27.96 & 5.78 \\
\hline 90 & 30.48 & 6.50 \\
\hline 100 & 32.84 & 7.23 \\
\hline 110 & 35.04 & 7.95 \\
\hline 120 & 37.10 & 8.67 \\
\hline 130 & 39.02 & 9.39 \\
\hline 140 & 40.82 & 10.12 \\
\hline 150 & 42.51 & 10.84 \\
\hline 160 & 44.09 & 11.56 \\
\hline
\end{tabular}

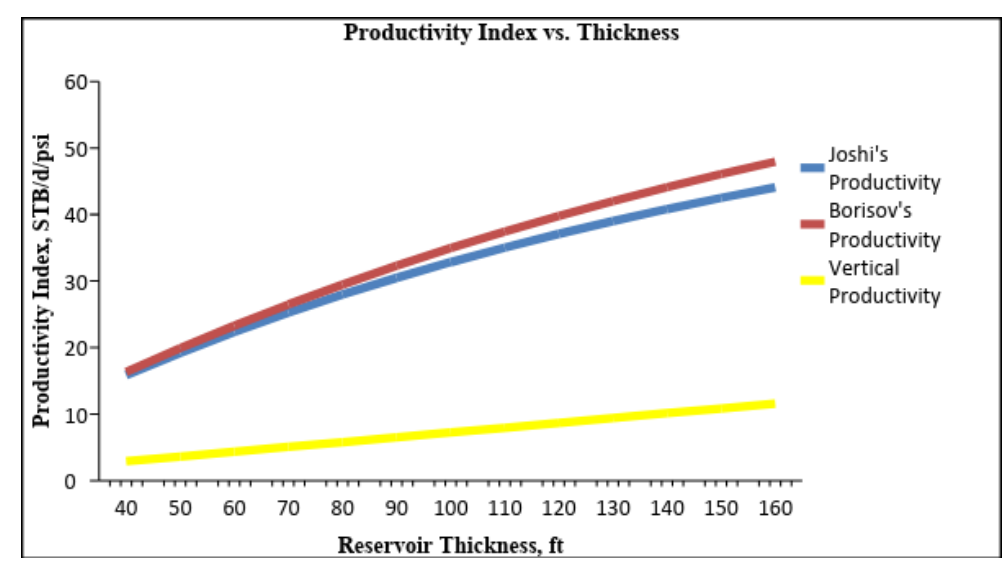

Figure4. Productivity Index versus Thickness 
Table6. Hypothetical Data for NPV Calculation

\begin{tabular}{|l|l|l|}
\hline \multicolumn{1}{|c|}{ Parameter } & \multicolumn{1}{c|}{ Value } & \multicolumn{1}{c|}{ Units } \\
\hline Annual decline rate & 10 & $\%$ \\
\hline Discount rate of return & 15 & $\%$ \\
\hline Opex/year & $15,000,000$ & $\$$ \\
\hline Peak production horizontal well & 5000 & $\mathrm{bbl} /$ day \\
\hline Exploration cost & $100,000,000$ & $\$$ \\
\hline Development cost (vertical well) & $65,000,000$ & $\$$ \\
\hline Development cost (horizontal well) & $110,000,000$ & $\$$ \\
\hline Tax Percentage & 30 & $\%$ \\
\hline Royalty & 12 & $\%$ \\
\hline Peak production vertical well & 2900 & $\mathrm{bbl} /$ day \\
\hline Initial pressure & 2850 & Psi \\
\hline Well bore pressure & 2790 & Psi \\
\hline
\end{tabular}

Table7. Horizontal Well Economic Evaluation (Borisov)

\begin{tabular}{|c|c|c|c|c|c|c|c|c|c|c|c|}
\hline Time & $\begin{array}{l}\text { Instanta } \\
\text { neous } \\
\text { Producti } \\
\text { on Bopd }\end{array}$ & $\begin{array}{c}\text { Yearly } \\
\text { Product } \\
\text { ion } \\
\text { (STB/ye } \\
\text { ar) } \\
\end{array}$ & $\begin{array}{l}\text { Gross } \\
\text { Reven } \\
\text { ue (\$) }\end{array}$ & $\begin{array}{c}\text { Royalt } \\
\text { y (\$) }\end{array}$ & $\begin{array}{c}\text { Net } \\
\text { Reven } \\
\text { ue (\$) }\end{array}$ & $\begin{array}{c}\text { CAPE } \\
X(\$)\end{array}$ & $\begin{array}{c}\text { OPEX } \\
(\$)\end{array}$ & $\begin{array}{l}\text { Taxable } \\
\text { Income } \\
(\$)\end{array}$ & $\begin{array}{c}30 \% \\
\text { Tax } \\
(\$)\end{array}$ & 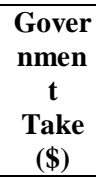 & $\begin{array}{c}\text { Oper } \\
\text { ator } \\
\text { Take } \\
(\$)\end{array}$ \\
\hline 2014 & 2876.38 & $\begin{array}{c}1209349 \\
.25\end{array}$ & $\begin{array}{c}133,02 \\
8,417 . \\
29\end{array}$ & $\begin{array}{c}15,963 \\
, 410.0 \\
7\end{array}$ & $\begin{array}{c}117,06 \\
5,007 . \\
21\end{array}$ & $\begin{array}{l}50,000, \\
000.00\end{array}$ & $\begin{array}{c}10,000 \\
, 000.0 \\
0\end{array}$ & $\begin{array}{c}57,065,00 \\
7.21\end{array}$ & $\begin{array}{c}17,119 \\
, 502.1 \\
6\end{array}$ & $\begin{array}{c}33,08 \\
2,912 . \\
24\end{array}$ & $\begin{array}{c}39,94 \\
5,505 . \\
05\end{array}$ \\
\hline 2015 & 3792.35 & $\begin{array}{c}1594460 \\
.97\end{array}$ & $\begin{array}{c}175,39 \\
0,707 . \\
20\end{array}$ & $\begin{array}{c}21,046 \\
, 884.8 \\
6\end{array}$ & $\begin{array}{c}154,34 \\
3,822 . \\
34\end{array}$ & $\begin{array}{l}50,000, \\
000.00\end{array}$ & $\begin{array}{c}10,000 \\
, 000.0 \\
0\end{array}$ & $\begin{array}{c}94,343,82 \\
2.34\end{array}$ & $\begin{array}{c}28,303 \\
, 146.7 \\
0\end{array}$ & $\begin{array}{c}49,35 \\
0,031 . \\
57\end{array}$ & $\begin{array}{c}66,04 \\
0,675 . \\
64\end{array}$ \\
\hline 2016 & 5000.00 & $\begin{array}{c}1825000 \\
.00\end{array}$ & $\begin{array}{c}200,75 \\
0,000 . \\
00\end{array}$ & $\begin{array}{c}24,090 \\
, 000.0 \\
0\end{array}$ & $\begin{array}{c}176,66 \\
0,000 . \\
00\end{array}$ & $\begin{array}{l}65,000, \\
000.00\end{array}$ & $\begin{array}{c}10,000 \\
, 000.0 \\
0\end{array}$ & $\begin{array}{c}101,660,0 \\
00.00\end{array}$ & $\begin{array}{c}30,498 \\
, 000.0 \\
0\end{array}$ & $\begin{array}{c}54,58 \\
8,000 . \\
00\end{array}$ & $\begin{array}{c}71,16 \\
2,000 . \\
00\end{array}$ \\
\hline 2017 & 4500.00 & $\begin{array}{c}1558933 \\
.14\end{array}$ & $\begin{array}{c}171,48 \\
2,645 . \\
92\end{array}$ & $\begin{array}{c}20,577 \\
, 917.5 \\
1\end{array}$ & $\begin{array}{c}150,90 \\
4,728 . \\
41\end{array}$ & $\begin{array}{l}45,000, \\
000.00\end{array}$ & $\begin{array}{c}10,000 \\
, 000.0 \\
0\end{array}$ & $\begin{array}{c}95,904,72 \\
8.41\end{array}$ & $\begin{array}{c}28,771 \\
, 418.5 \\
2\end{array}$ & $\begin{array}{c}49,34 \\
9,336 . \\
03\end{array}$ & $\begin{array}{c}67,13 \\
3,309 . \\
88\end{array}$ \\
\hline 2018 & 4050.00 & $\begin{array}{c}1403039 \\
.83\end{array}$ & $\begin{array}{c}154,33 \\
4,381 . \\
32\end{array}$ & $\begin{array}{c}18,520 \\
, 125.7 \\
6\end{array}$ & $\begin{array}{c}135,81 \\
4,255 . \\
56\end{array}$ & & $\begin{array}{c}10,000 \\
, 000.0 \\
0\end{array}$ & $\begin{array}{c}125,814,2 \\
55.56\end{array}$ & $\begin{array}{c}37,744 \\
, 276.6 \\
7\end{array}$ & $\begin{array}{c}56,26 \\
4,402 . \\
43\end{array}$ & $\begin{array}{c}88,06 \\
9,978 . \\
90\end{array}$ \\
\hline 2019 & 3645.00 & $\begin{array}{c}1262735 \\
.85\end{array}$ & $\begin{array}{c}138,90 \\
0,943 . \\
19\end{array}$ & $\begin{array}{c}16,668 \\
, 113.1 \\
8 \\
\end{array}$ & $\begin{array}{c}122,23 \\
2,830 . \\
01\end{array}$ & & $\begin{array}{c}10,000 \\
, 000.0 \\
0\end{array}$ & $\begin{array}{c}112,232,8 \\
30.01\end{array}$ & $\begin{array}{c}33,669 \\
, 849.0 \\
0\end{array}$ & $\begin{array}{c}50,33 \\
7,962 . \\
19\end{array}$ & $\begin{array}{c}78,56 \\
2,981 . \\
01\end{array}$ \\
\hline 2020 & 3280.50 & $\begin{array}{c}1136462 \\
.26\end{array}$ & $\begin{array}{c}125,01 \\
0,848 . \\
87\end{array}$ & $\begin{array}{c}15,001 \\
, 301.8 \\
6\end{array}$ & $\begin{array}{c}110,00 \\
9,547 . \\
01\end{array}$ & & $\begin{array}{c}10,000 \\
, 000.0 \\
0\end{array}$ & $\begin{array}{c}100,009,5 \\
47.01\end{array}$ & $\begin{array}{c}30,002 \\
, 864.1 \\
0\end{array}$ & $\begin{array}{c}45,00 \\
4,165 . \\
97\end{array}$ & $\begin{array}{c}70,00 \\
6,682 . \\
91\end{array}$ \\
\hline 2021 & 2952.45 & $\begin{array}{c}1022816 \\
0.36\end{array}$ & $\begin{array}{c}1,125, \\
097,63 \\
9.85\end{array}$ & $\begin{array}{c}135,01 \\
1,716 . \\
78\end{array}$ & $\begin{array}{c}990,08 \\
5,923 . \\
07\end{array}$ & & $\begin{array}{c}10,000 \\
, 000.0 \\
0\end{array}$ & $\begin{array}{c}980,085,9 \\
23.07\end{array}$ & $\begin{array}{c}294,02 \\
5,776 . \\
92\end{array}$ & $\begin{array}{c}429,0 \\
37,49 \\
3.70\end{array}$ & $\begin{array}{c}686,0 \\
60,14 \\
6.15\end{array}$ \\
\hline & Total & & $\begin{array}{c}2,223, \\
995,58 \\
3.64\end{array}$ & & & & \multicolumn{4}{|c|}{ NPV (Operator) } & $\begin{array}{c}498,1 \\
88,99 \\
1.57\end{array}$ \\
\hline & & & & & & & \multicolumn{4}{|c|}{ NPV (Government) } & $\begin{array}{c}337,0 \\
99,29 \\
6\end{array}$ \\
\hline
\end{tabular}

Table8. Horizontal Well Economic Evaluation (Joshi)

\begin{tabular}{|c|c|c|c|c|c|c|c|c|c|c|c|}
\hline Time & $\begin{array}{c}\text { Instant } \\
\text { aneous } \\
\text { Product } \\
\text { ion } \\
\text { Bopd }\end{array}$ & $\begin{array}{c}\text { Yearly } \\
\text { Product } \\
\text { ion } \\
\text { (STB/ye } \\
\text { ar) }\end{array}$ & $\begin{array}{c}\text { Gross } \\
\text { Reven } \\
\text { ue }(\$)\end{array}$ & $\begin{array}{c}\text { Royalty } \\
(\$)\end{array}$ & $\begin{array}{c}\text { Net } \\
\text { Revenu } \\
\text { e }(\$)\end{array}$ & $\begin{array}{c}\text { CAP } \\
\text { EX } \\
(\$)\end{array}$ & $\begin{array}{c}\text { OPEX } \\
(\$)\end{array}$ & $\begin{array}{c}\text { Taxable } \\
\text { Income } \\
(\$)\end{array}$ & $\begin{array}{c}\mathbf{3 0 \%} \\
\text { Tax } \\
(\$)\end{array}$ & $\begin{array}{c}\text { Gover } \\
\text { nment } \\
\text { Take } \\
\text { (\$) }\end{array}$ & $\begin{array}{c}\text { Opera } \\
\text { tor's } \\
\text { Take } \\
(\$)\end{array}$ \\
\hline 2014 & 2645.56 & $\begin{array}{c}111230 \\
2.43\end{array}$ & $\begin{array}{c}122,35 \\
3,267 \\
27\end{array}$ & $\begin{array}{l}14,682, \\
392.07\end{array}$ & $\begin{array}{c}107,670, \\
875.20\end{array}$ & $\begin{array}{l}50,00 \\
0,000\end{array}$ & $\begin{array}{c}10,000 \\
, 000\end{array}$ & $\begin{array}{c}47,670,8 \\
75.20\end{array}$ & $\begin{array}{c}14,301 \\
, 262.5 \\
6\end{array}$ & $\begin{array}{c}28,983 \\
654.63\end{array}$ & $\begin{array}{c}33,369 \\
, 612.6 \\
4\end{array}$ \\
\hline 2015 & 3488.02 & $\begin{array}{c}199625 \\
9.61\end{array}$ & $\begin{array}{c}219,58 \\
8,556 . \\
64\end{array}$ & $\begin{array}{c}26,350 \\
626.80\end{array}$ & $\begin{array}{c}193,237, \\
929.84\end{array}$ & $\begin{array}{l}50,00 \\
0,000\end{array}$ & $\begin{array}{c}10,000 \\
, 000\end{array}$ & $\begin{array}{c}133,237 \\
929.84\end{array}$ & $\begin{array}{c}39,971 \\
378.9 \\
5\end{array}$ & $\begin{array}{l}66,322 \\
005.75\end{array}$ & $\begin{array}{c}93,266 \\
550.8 \\
9\end{array}$ \\
\hline 2016 & 5000.00 & $\begin{array}{c}182500 \\
0.00\end{array}$ & $\begin{array}{c}200,75 \\
0,000 . \\
00\end{array}$ & $\begin{array}{c}24,090 \\
000.00\end{array}$ & $\begin{array}{c}176,660 \\
000.00\end{array}$ & $\begin{array}{l}65,00 \\
0,000\end{array}$ & $\begin{array}{c}10,000 \\
, 000\end{array}$ & $\begin{array}{c}101,660 \\
000.00\end{array}$ & $\begin{array}{c}30,498 \\
, 000.0 \\
0\end{array}$ & $\begin{array}{c}54,588 \\
000.00\end{array}$ & $\begin{array}{c}71,162 \\
, 000.0 \\
0\end{array}$ \\
\hline
\end{tabular}


Comparison of the Economics and Performance of Horizontal and Vertical Wells

\begin{tabular}{|c|c|c|c|c|c|c|c|c|c|c|c|}
\hline 2017 & 4500.00 & $\begin{array}{c}155893 \\
3.14\end{array}$ & $\begin{array}{c}171,48 \\
2,645 . \\
92\end{array}$ & $\begin{array}{l}20,577, \\
917.51\end{array}$ & $\begin{array}{c}150,904, \\
728.41\end{array}$ & $\begin{array}{l}45,00 \\
0,000\end{array}$ & $\begin{array}{c}10,000 \\
, 000\end{array}$ & $\begin{array}{c}95,904,7 \\
28.41\end{array}$ & $\begin{array}{c}28,771 \\
418.5 \\
2\end{array}$ & $\begin{array}{l}49,349, \\
336.03\end{array}$ & $\begin{array}{c}67,133 \\
, 309.8 \\
8\end{array}$ \\
\hline 2018 & 4050.00 & $\begin{array}{c}140303 \\
9.83\end{array}$ & $\begin{array}{c}154,33 \\
4,381 . \\
32\end{array}$ & $\begin{array}{l}18,520, \\
125.76\end{array}$ & $\begin{array}{c}135,814, \\
255.56\end{array}$ & & $\begin{array}{c}10,000 \\
, 000\end{array}$ & $\begin{array}{c}125,814 \\
255.56\end{array}$ & $\begin{array}{c}37,744 \\
, 276.6 \\
7\end{array}$ & $\begin{array}{l}56,264, \\
402.43\end{array}$ & $\begin{array}{c}88,069 \\
, 978.9 \\
0\end{array}$ \\
\hline 2019 & 3645.00 & $\begin{array}{c}126273 \\
5.85\end{array}$ & $\begin{array}{c}138,90 \\
0,943 . \\
19\end{array}$ & $\begin{array}{l}16,668, \\
113.18\end{array}$ & $\begin{array}{c}122,232, \\
830.01\end{array}$ & & $\begin{array}{c}10,000 \\
, 000\end{array}$ & $\begin{array}{c}112,232, \\
830.01\end{array}$ & $\begin{array}{c}33,669 \\
, 849.0 \\
0\end{array}$ & $\begin{array}{l}50,337, \\
962.19\end{array}$ & $\begin{array}{c}78,562 \\
, 981.0 \\
1\end{array}$ \\
\hline 2020 & 3280.50 & $\begin{array}{c}113646 \\
2.26\end{array}$ & $\begin{array}{c}125,01 \\
0,848 . \\
87\end{array}$ & $\begin{array}{l}15,001, \\
301.86\end{array}$ & $\begin{array}{c}110,009, \\
547.01\end{array}$ & & $\begin{array}{c}10,000 \\
, 000\end{array}$ & $\begin{array}{c}100,009, \\
547.01\end{array}$ & $\begin{array}{c}30,002 \\
, 864.1 \\
0\end{array}$ & $\begin{array}{l}45,004, \\
165.97\end{array}$ & $\begin{array}{c}70,006 \\
, 682.9 \\
1\end{array}$ \\
\hline 2021 & 2952.45 & $\begin{array}{c}102281 \\
60.36\end{array}$ & $\begin{array}{c}1,125 \\
097,63 \\
9\end{array}$ & $\begin{array}{c}135,011 \\
, 716\end{array}$ & $\begin{array}{c}990,085, \\
923.07\end{array}$ & & $\begin{array}{c}10,000 \\
, 000\end{array}$ & $\begin{array}{c}980,085, \\
923.07\end{array}$ & $\begin{array}{c}294,02 \\
5,776 . \\
92\end{array}$ & $\begin{array}{c}429,03 \\
7,493.7 \\
0\end{array}$ & $\begin{array}{c}686,06 \\
0,146 . \\
15\end{array}$ \\
\hline & Total & & $\begin{array}{c}2,257, \\
518,28 \\
3.06\end{array}$ & & & & \multicolumn{4}{|c|}{ NPV (Operator) } & $\begin{array}{c}513,05 \\
7,497 . \\
42\end{array}$ \\
\hline & & & & & & & \multicolumn{4}{|c|}{ NPV (Government) } & $\begin{array}{c}346,36 \\
7,974 . \\
49\end{array}$ \\
\hline
\end{tabular}

Table9. Economics of a Vertical Well

\begin{tabular}{|c|c|c|c|c|c|c|c|c|c|c|c|}
\hline Time & $\begin{array}{c}\text { Instant } \\
\text { aneous } \\
\text { Product } \\
\text { ion } \\
\text { Bopd }\end{array}$ & $\begin{array}{l}\text { Yearly } \\
\text { Product } \\
\text { ion } \\
\text { (STB/ye } \\
\text { ar) }\end{array}$ & $\begin{array}{l}\text { Gross } \\
\text { Reven } \\
\text { ue (\$) }\end{array}$ & $\begin{array}{c}\text { Royalt } \\
\text { y (\$) }\end{array}$ & $\begin{array}{c}\text { Net } \\
\text { Revenu } \\
\text { e (\$) }\end{array}$ & $\begin{array}{c}\text { CAP } \\
\text { EX } \\
(\$)\end{array}$ & $\begin{array}{l}\text { OPE } \\
\text { X (\$) }\end{array}$ & $\begin{array}{c}\text { Taxable } \\
\text { Income } \\
(\$)\end{array}$ & $\begin{array}{c}30 \% \\
\operatorname{Tax}(\$)\end{array}$ & $\begin{array}{c}\text { Govern } \\
\text { ment } \\
\text { Take } \\
(\$)\end{array}$ & 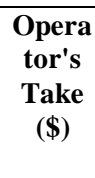 \\
\hline 2014 & 693.76 & $\begin{array}{c}956759 . \\
99\end{array}$ & $\begin{array}{c}105,24 \\
3,598 . \\
68\end{array}$ & $\begin{array}{c}12,629 \\
, 231.8 \\
4\end{array}$ & $\begin{array}{l}92,614, \\
366.84\end{array}$ & $\begin{array}{l}50,00 \\
0,000\end{array}$ & $\begin{array}{l}10,00 \\
0,000\end{array}$ & $\begin{array}{c}32,614,3 \\
66.84\end{array}$ & $\begin{array}{c}9,784,31 \\
0.05\end{array}$ & $\begin{array}{c}22,413,5 \\
41.89\end{array}$ & $\begin{array}{c}22,830 \\
, 056.7 \\
9\end{array}$ \\
\hline 2015 & 1418.42 & $\begin{array}{c}195612 \\
2.07\end{array}$ & $\begin{array}{c}215,17 \\
3,428 . \\
21\end{array}$ & $\begin{array}{c}25,820 \\
, 811.3 \\
9\end{array}$ & $\begin{array}{l}189,352 \\
, 616.82\end{array}$ & $\begin{array}{l}50,00 \\
0,000\end{array}$ & $\begin{array}{l}10,00 \\
0,000\end{array}$ & $\begin{array}{c}129,352 \\
616.82\end{array}$ & $\begin{array}{c}38,805,7 \\
85.05\end{array}$ & $\begin{array}{c}64,626,5 \\
96.43\end{array}$ & $\begin{array}{c}90,546 \\
, 831.7 \\
8\end{array}$ \\
\hline 2016 & 2900.00 & $\begin{array}{c}105850 \\
0.00\end{array}$ & $\begin{array}{c}116,43 \\
5,000 . \\
00\end{array}$ & $\begin{array}{c}13,972 \\
, 200.0 \\
0\end{array}$ & $\begin{array}{l}102,462 \\
, 800.00\end{array}$ & $\begin{array}{l}35,00 \\
0,000\end{array}$ & $\begin{array}{l}10,00 \\
0,000\end{array}$ & $\begin{array}{c}57,462,8 \\
00.00\end{array}$ & $\begin{array}{c}17,238,8 \\
40.00\end{array}$ & $\begin{array}{c}31,211,0 \\
40.00\end{array}$ & $\begin{array}{c}40,223 \\
, 960.0 \\
0\end{array}$ \\
\hline 2017 & 2610.00 & $\begin{array}{c}904181 . \\
22\end{array}$ & $\begin{array}{c}99,459 \\
, 934.6 \\
3\end{array}$ & $\begin{array}{c}11,935 \\
, 192.1 \\
6\end{array}$ & $\begin{array}{l}87,524, \\
742.48\end{array}$ & $\begin{array}{l}30,00 \\
0,000\end{array}$ & $\begin{array}{l}10,00 \\
0,000\end{array}$ & $\begin{array}{c}47,524,7 \\
42.48\end{array}$ & $\begin{array}{c}14,257,4 \\
22.74\end{array}$ & $\begin{array}{c}26,192,6 \\
14.90\end{array}$ & $\begin{array}{c}33,267 \\
, 319.7 \\
3\end{array}$ \\
\hline 2018 & 2349.00 & $\begin{array}{c}813763 . \\
10\end{array}$ & $\begin{array}{c}89,513 \\
, 941.1 \\
7\end{array}$ & $\begin{array}{c}10,741 \\
, 672.9 \\
4\end{array}$ & $\begin{array}{l}78,772, \\
268.23\end{array}$ & & $\begin{array}{l}10,00 \\
0,000\end{array}$ & $\begin{array}{c}68,772,2 \\
68.23\end{array}$ & $\begin{array}{c}20,631,6 \\
80.47\end{array}$ & $\begin{array}{c}31,373,3 \\
53.41\end{array}$ & $\begin{array}{c}48,140 \\
, 587.7 \\
6\end{array}$ \\
\hline 2019 & 2114.10 & $\begin{array}{c}732386 . \\
79\end{array}$ & $\begin{array}{c}80,562 \\
, 547.0 \\
5\end{array}$ & $\begin{array}{l}9,667, \\
505.65\end{array}$ & $\begin{array}{l}70,895, \\
041.40\end{array}$ & & $\begin{array}{l}10,00 \\
0,000\end{array}$ & $\begin{array}{c}60,895,0 \\
41.40\end{array}$ & $\begin{array}{c}18,268,5 \\
12.42\end{array}$ & $\begin{array}{c}27,936,0 \\
18.07\end{array}$ & $\begin{array}{c}42,626 \\
, 528.9 \\
8\end{array}$ \\
\hline 2020 & 1902.69 & $\begin{array}{c}659148 . \\
11\end{array}$ & $\begin{array}{c}72,506 \\
, 292.3 \\
5\end{array}$ & $\begin{array}{l}8,700, \\
755.08\end{array}$ & $\begin{array}{l}63,805, \\
537.26\end{array}$ & & $\begin{array}{l}10,00 \\
0,000\end{array}$ & $\begin{array}{c}53,805,5 \\
37.26\end{array}$ & $\begin{array}{c}16,141,6 \\
61.18\end{array}$ & $\begin{array}{c}24,842,4 \\
16.26\end{array}$ & $\begin{array}{c}37,663 \\
, 876.0 \\
9\end{array}$ \\
\hline 2021 & 1712.42 & $\begin{array}{c}593233 \\
3.01\end{array}$ & $\begin{array}{c}652,55 \\
6,631 . \\
11\end{array}$ & $\begin{array}{c}78,306 \\
, 795.7 \\
3\end{array}$ & $\begin{array}{r}574,249 \\
, 835.38\end{array}$ & & $\begin{array}{l}10,00 \\
0,000\end{array}$ & $\begin{array}{c}564,249, \\
835.38\end{array}$ & $\begin{array}{c}169,274 \\
950.6\end{array}$ & $\begin{array}{c}247,581, \\
746.35\end{array}$ & $\begin{array}{c}394,97 \\
4,884 . \\
77\end{array}$ \\
\hline \multicolumn{3}{|c|}{ Total } & $\begin{array}{c}1,431, \\
451,37 \\
3.2\end{array}$ & & & & \multicolumn{4}{|c|}{ NPV (Operator) } & $\begin{array}{c}319,42 \\
7,483 . \\
00\end{array}$ \\
\hline & & & & & & & \multicolumn{4}{|c|}{ NPV (Government) } & $\begin{array}{c}\$ 221,8 \\
04,215 \\
.5\end{array}$ \\
\hline
\end{tabular}

\subsection{Discussion}

\subsubsection{Effects of Well Length on Horizontal Productivity}

The results of productivity index using Borisov and Joshi's models are summarized in Table 2 and Table 3 respectively. All calculations were done using Microsoft Excel software.

The different values of calculated productivity ratios from two different horizontal well productivity models were plotted against varying well lengths of the perforated horizontal section. Figure 3 is a graph representing such a plot.

It can be observed from the graph that the productivity index of horizontal wells increases as the length of the perforated horizontal section of the well is increased. A length of $250 \mathrm{ft}$ registers a 
productivity ratio of 1.58 and 1.40 from Borisov and Joshi's model respectively. A length of $750 \mathrm{ft}$, $1250 \mathrm{ft}$, and $2000 \mathrm{ft}$ registered productivity ratios of 3.34, 4.99, and 8.09 respectively for Borisov's model and 3.05, 460, and 7.32 respectively for Joshi's model.

No change was observed in the productivity index of a vertical well calculated using Joshi's model. The vertical well productivity index was calculated to be $11.56 \mathrm{STB} /$ day/psi using Joshi's model for vertical productivity index. The vertical productivity remained $11.56 \mathrm{STB} / \mathrm{day} / \mathrm{psi}$ because the well length (L) parameter is not accounted for in Joshi's equation for vertical productivity.

\subsubsection{Effects of Reservoir Thickness on well Productivity}

The parameter of reservoir thickness (h) was varied in the various equations for calculation of productivity index, starting from $40 \mathrm{ft}$. to $160 \mathrm{ft}$. while maintaining a horizontal well length section of $1000 \mathrm{ft}$. Both horizontal and vertical productivity indices were observed to increase with increasing reservoir thickness. Table 4 and table 5 are summaries of the calculations involving variations in reservoir thickness.

The vertical productivity index was calculated to be $2.89 \mathrm{STB} /$ day/psi, $5.78 \mathrm{STB} /$ day/psi, 8.67 $\mathrm{STB} / \mathrm{day} / \mathrm{psi}$, and $11.56 \mathrm{STB} / \mathrm{day} / \mathrm{psi}$ at reservoir thickness of $40 \mathrm{ft}, 80 \mathrm{ft}, 120 \mathrm{ft}$, and $160 \mathrm{ft}$ respectively. Using Borisov's model, productivity index values of $16.35 \mathrm{STB} / \mathrm{day} / \mathrm{psi}, 29.47$ $\mathrm{STB} / \mathrm{day} / \mathrm{psi}, 39.79 \mathrm{STB} / \mathrm{day} / \mathrm{psi}$, and $47.94 \mathrm{STB} /$ day/psi were calculated with corresponding reservoir thicknesses of $40 \mathrm{ft}, 80 \mathrm{ft}, 120 \mathrm{ft}$, and $160 \mathrm{ft}$ respectively.

Productivity index values of 15.86 STB/day/psi, 27.96 STB/day/psi, 37.10 STB/day/psi, and 44.09 $\mathrm{STB} /$ day/psi were calculated using Joshi's model with corresponding reservoir thickness of $40 \mathrm{ft}, 80$ $\mathrm{ft}, 120 \mathrm{ft}$, and $160 \mathrm{ft}$ respectively. A graph of productivity index against reservoir thickness was plotted in figure 3.4 to show graphical representations of how reservoir thickness affects productivity.

\subsubsection{Net Present Value}

A summary of the net present value calculations are shown in table 3.7, table 3.8 and table 3.9. From Table 3.7, the Net Present Value of the operator's take of the horizontal well using Borisov's horizontal productivity index for production forecast was $\$ 498,188,991.57$, while the Net Present Value of the government's take was $\$ 337,099,296$.

The Net Present Value of the operator's take from the horizontal well using Joshi's productivity index for production forecast was $\$ 513,057,497.42$, while the Net Present Value of government's take was $\$ 346,367,974.49$.

Using the vertical productivity index to forecast production from the vertical well, a Net Present Value of $\$ 319,427,483.00$ was calculated from the operator's take, while a Net Present Value Of $\$ 221,804,215.51$ was calculated from the government's take.

It is therefore evident that the horizontal wells in this project are more economical to both the operator and the host government than the vertical well.

\section{CONClusion}

The following conclusions can be drawn from this study;

- Horizontal wells have a higher productivity than a conventional vertical well.

- Horizontal well productivity index increases with increasing horizontal well length while vertical productivity index is not affected by well length.

- Both horizontal and vertical productivity indices increase with increasing reservoir thickness.

- Sinking a horizontal well in this case is more economical than sinking a vertical well as its net present value is greater than the net present value of a vertical well.

\section{ACKNOWLEDGEMENT}

I would like to appreciate the University of Mines and Technology (UMaT) - Tarkwa for their support. 


\section{REFERENCES}

[1] Dankwa O. K. and Igbokoyi A. O., "Effects of Partial Completion on Productivity Index", Nigeria Annual International Conference and Exhibition, Lagos, 6-8 August, Nigeria, (2012).

[2] Ibelegbu, C. "Productivity Index in Horizontal Wells", Journal of Scientific and Industrial Research, Vol. 63, pp. $979-984$, (2004).

[3] Ibragimov, A., Khalmanova, D., Valko, P. P. and Walton, J. R. "On A Mathematical Model of The Productivity Index Of A Well From Reservoir Engineering”, Society for Industrial and Applied Mathematics, Vol. 65, No. 6, pp. 1952-1980, (2005).

[4] Lu, J. and Tiab, D. "Pseudo-Steady-State Productivity Formula for a Partially Penetrating Vertical Well in a Box-Shaped Reservoir", Mathematical Problems in Engineering, Vol. 2010, Article ID 907206, 35pp, (2010).

[5] Kareem, L. A., Sultan, A. S. And Iwalewa, T. M. "A simplified semi-analytical productivity index for a horizontal well with a non-linear trajectory in a vertical plane", Energy Exploration \& Exploitation, Vol. 33, No. 6, pp. 785-808, (2015).

[6] Zhang, L. "Analysis of Fluid Flow to Horizontal and Slant Wells", Unpublished PhD Dissertation, University of Waterloo, 190pp, (1997).

[7] Proett, M. A., Ansah, J., Soliman, M. Y., Schultz, R. and Folse, K. "Productivity Optimization of Oil Wells Using a New 3D Finite-Element Wellbore Inflow Model and Artificial Neural Network" International ANSYS Conference, May 24-26, Pittsburgh, PA, USA, 17pp, (2004).

[8] Oaikhena, E. E. and Oloro, J. "A Comparative Study of the Productivity Index of Horizontal Well", Greener Journal of Physical Sciences, Vol. 3, pp. 97 - 109, (2013).

[9] Guido, B., Pete, L. and Mike, W. "Drilling Straight Down”, Oil Review, pp. 14 - 17, (2004).

[10] Pan, S. "Stability Analysis of the Rotary Drill-String", Unpublished PhD Dissertation, University of Tennessee, Knoxville, 143pp, (2014).

[11] Campero, C. P. F. "Technology and Economics Affecting Unconventional Reservoir Development", Unpublished MSc Thesis, Texas A\&M University, USA, 223pp, (2008).

[12] Tabatabaei, M., Ghalabor, S. "A New Method to Predict Performance of Horizontal and Multilateral Wells", International Petroleum Technology Conference, Doha, Qatar, pp. 75 - 87, (2011).

[13] David, S. and Appah, D. "A Software for Productivity Enhancement of Horizontal Wells", Journal of Scientific and Engineering Research, 4(3):15-25, (2017).

[14] Borisov, J. P., 1964, "Oil Production Using Horizontal and Multiple Deviation Wells", Nedra, Moscow, Translated by J.Stauss and S.D.Joshi, eds., Phillips Petroleum Co., the R\&D Library Translation, Bartlesville, Oklahoma, (1984).

[15] Joshi, S. D. "Horizontal Well Technology”, Pennwell Publishing Company, Tulsa, Oklahoma pp. 34 - 47, (1991).

[16] Tarek, A. "Horizontal Well Performance", Reservoir Engineering Handbook $2^{\text {nd }}$ Edition, Gulf Publishing Company, Houston, Texas, pp. 515-526, (2006).

\section{AUTHORS' BIOGRAPHY}

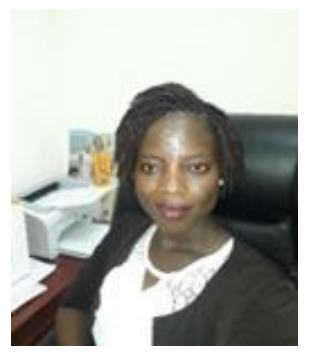

Dr Ohenewaa Kakra Dankwa, is a Lecturer at the Petroleum Engineering Department of the University of Mines and Technology (UMaT). She holds a BSc in Geomatic Engineering from the University of Mines and Technology (UMaT), Ghana. She obtained her MSc in Petroleum Engineering from the African University of Science and Technology (AUST) and Doctor of Philosophy $(\mathrm{PhD})$ in Petroleum Engineering from the University of Port Harcourt under the World Bank Africa Center of Excellence (ACE). Her research interests include productivity enhancement with matrix acidizing fluids, oilfields scales, environmental management, health and safety.

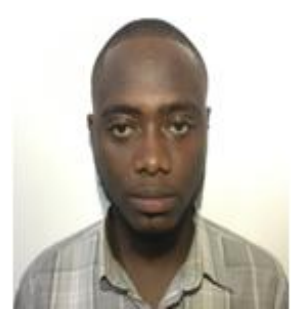

Salim Nubalanaang Gomah Ahmed, is a field officer at the Department of Petroleum Product Marking Scheme (PPMS) of the National Petroleum Authority (NPA), Ghana. His work is to ensure the quality, integrity, and standards of petroleum products and services provided by the service providers in the country. He also holds a BSc degree in Petroleum Engineering from the University of Mines and Technology (UMaT), Tarkwa, Ghana. His research interest is in drilling operations, specifically drilling vessels. 


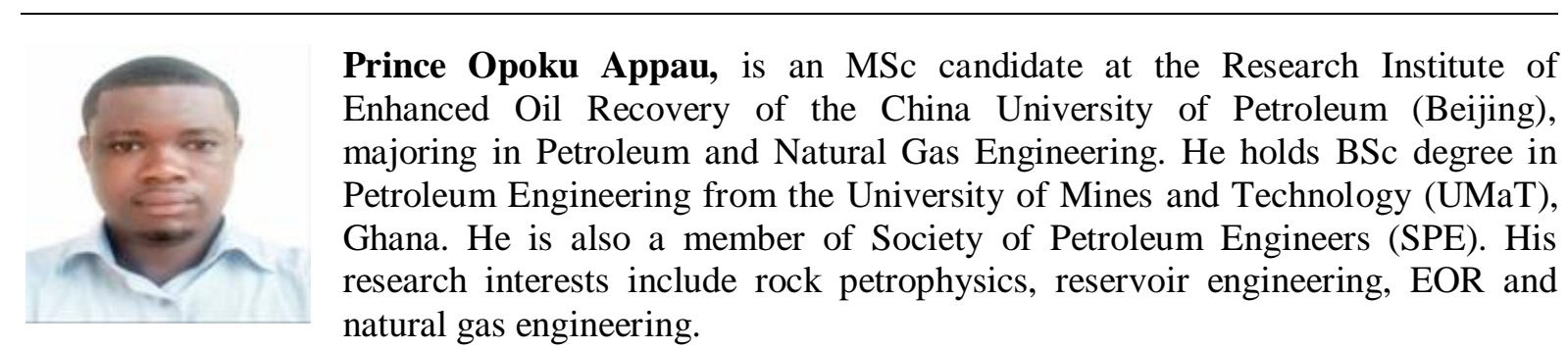

Citation: O. K. Dankwa, et.al. (2018). Comparison of the Economics and Performance of Horizontal and Vertical Wells, International Journal of Petroleum and Petrochemical Engineering (IJPPE), 4(3), pp.1-12, DOI: http://dx.doi.org/10.20431/2454-7980.0403001

Copyright: (C) 2018 O. K. Dankwa. This is an open-access article distributed under the terms of the Creative Commons Attribution License, which permits unrestricted use, distribution, and reproduction in any medium, provided the original author and source are credited 